\title{
Citrobacter rodentium: a model enteropathogen for understanding the interplay of innate and adaptive components of type 3 immunity
}

\author{
DJ Silberger ${ }^{1}, \mathrm{CL}$ Zindl $^{1}$ and CT Weaver ${ }^{1}$
}

Citrobacter rodentium is a natural murine intestinal pathogen that shares a core set of virulence factors with the related human pathogens enteropathogenic Escherichia coli (EPEC) and enterohemorrhagic E. coli (EHEC). C. rodentium is now the most widely used small animal model for studying the molecular underpinnings of EPEC and EHEC infections in vivo, including: enterocyte attachment; virulence; colonization resistance; and mucosal immunity. In this review, we discuss type 3 immunity in the context of $C$. rodentium infection and discuss recent publications that use this model to understand how the innate and adaptive components of immunity intersect to mediate host protection against enteric pathogens and maintain homeostasis with the microbiota.

\section{HIGHLIGHTS}

- Citrobacter rodentium infection is an excellent model for studying the interactions between innate and adaptive lymphocytes that characterize type 3 immune responses to enteropathogens.

- Type 3 immune responses enable the clearance of C. rodentium through the collaboration between antigenpresenting cells, group 3 innate lymphoid cells, pathogenspecific $\mathrm{B}$ cells and $\mathrm{T}$ cells, and the epithelium.

- IL-22 protects the intestinal barrier from enteropathogens and reduces systemic dissemination of enterobacterial pathobionts by enhancing the expression of gap junction proteins, fucosylated mucins, bactericidal proteins, and complement C3.

- IL-21 expedites the clearance of C. rodentium independently of IL-22 in the later stages of infection.

GLOBAL BURDEN OF DIARRHEAGENIC ESCHERICHIA COLI Diarrheagenic pathogens cause $\sim 0.8$ million deaths per year in children under the age of 5 years and are the second leading cause of infection-related deaths in this demographic. ${ }^{1-3}$ While many enteric pathogens can cause diarrhea, pathogenic E. coli is a major contributor to diarrheal-related deaths. Diarrheagenic
E. coli are categorized into seven pathotypes that have been previously reviewed, including: enteropathogenic E. coli (EPEC), enterohemorrhagic E. coli (EHEC), enterotoxigenic E. coli (ETEC) enteroinvasive E. coli (EIEC), enteroaggregative E. coli (EAEC), diffusely adherent E. coli (DAEC), and adherent invasive E. coli (AIEC). ${ }^{4,5}$ Of note, infections with EPEC and ETEC are particularly lethal in developing countries. ${ }^{2}$ In contrast, infections with EPEC, which caused outbreaks in neonates in the United Kingdom and United States in the 1940s and 1950s, are no longer thought to be common in the clinical setting in these countries. ${ }^{5}$ Nevertheless, several diarrheagenic E. coli pathotypes that do not produce Shiga toxin are not reported by the $\mathrm{CDC}^{6}$ and their incidence is unknown. Importantly, the annual number of multistate outbreaks caused by Shiga toxin-producing $\left(\mathrm{Stx}^{+}\right)$EHEC in the United States increased from 1 to 10 between 1998 and 2014, and the largest global outbreak of diarrheagenic E. coli occurred in 2011 by a Stx ${ }^{+}$EAEC. ${ }^{4,7}$ EPEC and EHEC share a similar colonization strategy known as attaching and effacing $(\mathrm{A} / \mathrm{E})$ lesion formation. ${ }^{4,8} \mathrm{~A} / \mathrm{E}$ enteropathogens express conserved virulence genes in a genomic island termed the locus of enterocyte effacement (LEE); expression of LEE-encoded genes are required for successful colonization and pathogenicity. ${ }^{9}$ Furthermore, studies demonstrated that K12 strains of E. coli transformed with the LEE locus acquire the A/E phenotype,

${ }^{1}$ Department of Pathology, University of Alabama at Birmingham, Birmingham, Alabama, USA. Correspondence: CT Weaver (cweaver@uab.edu)

Received 30 September 2016; accepted 13 April 2017; published online 14 June 2017. doi:10.1038/mi.2017.47 
indicating that horizontal gene transfer of this pathogenicity island is sufficient for the virulence of these microorganisms. ${ }^{10}$ While significant progress has been made developing intestinal culture systems for studying these pathogens in vitro, transgenic mouse models provide powerful tools to assess the contributions of the mucosal immune system and microbiota in response to enteropathogenic infection. ${ }^{11-13}$

\section{CITROBACTER RODENTIUM INFECTION MODEL FOR ATTACHING AND EFFACING ENTEROPATHOGENS}

C. rodentium was identified as the etiological agent of transmissible murine colonic hyperplasia in a mouse colony outbreak and remains the only known naturally occurring A/E enteropathogen of mice. ${ }^{8}$ Because colonization of mice with EPEC and EHEC requires pretreatment with antibiotics, C. rodentium has become the principal rodent model for studying infections with $\mathrm{A} / \mathrm{E}$ enteropathogens. ${ }^{14,15}$ Additionally, a Stx-expressing strain of $C$. rodentium was recently generated and now offers a natural infection model for studying Shiga toxin-producing E. coli. ${ }^{16}$ Studies with C. rodentium deletion mutants were instrumental in identifying LEE-encoded and nonLEE-encoded genes that are critical for enteropathogen colonization and pathogenicity. ${ }^{17}$ The most widely studied of these virulence factors include the adhesin intimin, the type III secretion system, the translocator protein EspA, and the translocated intimin receptor. Importantly, several vaccine studies have reported success at reducing the colonization of $\mathrm{A} / \mathrm{E}$ enteropathogens by using intimin, translocated intimin receptor, and EspA as antigens in various host species. ${ }^{18,19}$ Additionally, passively transferred antibodies against EspA were shown to protect mice against infection with EHEC. ${ }^{20}$

C. rodentium primarily affects the distal large intestine, but the tissue tropism differs depending on the inoculation method. For example, when C. rodentium is passaged overnight in liquid culture, colonization begins at the cecal patch and descends toward the distal colon over subsequent days of infection. ${ }^{21}$ Interestingly, when mice are infected via natural transmission during cohousing with orally infected mice, $C$. rodentium bypasses the cecum, infection peaks earlier and requires $10^{3}$ fold fewer bacteria. ${ }^{21}$ Thus, infection with naturally transmitted C. rodentium occurs more efficiently, suggesting that cecal colonization is an adaptation to the intestinal environment during which the bacterium upregulates virulence gene expression. ${ }^{21-23}$ Several virulence factors that are induced during infection can be expressed in vitro by culturing the bacteria in Dulbecco's modified Eagle's medium, which activates ler, a global transcriptional regulator of several LEE-encoded genes. ${ }^{9,17,22}$ Recently, Kamada et al. ${ }^{22}$ designed a bioluminescent reporter $C$. rodentium strain in which the ler promoter was fused to the luxCDABE operon of Photorhabdus luminescens to report the expression of LEE-encoded virulence factors during C. rodentium infection. ${ }^{22}$ The ler-lux ${ }^{+}$ C. rodentium reporter and $\Delta l e r$ mutant were then used to demonstrate that LEE-encoded genes must be expressed for C. rodentium to compete with the microbiota in conventionally housed mice. ${ }^{24}$ In contrast, C. rodentium downregulates
LEE-encoded genes in germ-free mice and relocates to the intestinal lumen where it is outcompeted upon introduction of the microbiota from SPF mice or E. coli. ${ }^{22,24,25}$ Moreover, it was recently demonstrated that $C$. rodentium utilizes the type III secretion system and A/E lesion formation to increase local oxygenation at the mucosal surface, at least in part via stimulation of more rapid cell division of undifferentiated transit amplifying (TA) epithelial cells, which rely less on butyrate metabolism that is a primary energy source for superficial epithelial cells-and which depletes oxygen. ${ }^{26}$ This facilitates the aerobic respiration of $C$. rodentium to permit its more rapid growth while potentially restraining competition with anaerobic constituents of the microbiota that are impaired by increased oxygen. These studies suggest that $\mathrm{A} / \mathrm{E}$ lesion formation represents an evolutionary adaptation by C. rodentium to compete with the endogenous microbiota for colonization and transmission, and the distribution of colonization along the cecum-colonic axis may reflect geographical differences in the endogenous microbiota with which $C$. rodentium must compete.

C. rodentium attachment typically occurs on the apical surface of superficial enterocytes that line the intestinal lumen and does not extend to epithelial cells that line the sides and the base of crypts in wild-type mice. ${ }^{27}$ Whether this restricted pattern of attachment reflects differences in the expression of anti-microbial proteins, host receptors, goblet cells, or mucin fucosylation along the crypt axis, or is driven by the presence of innate and adaptive lymphocyte populations that reside in the lamina propria, isolated lymphoid follicles, or colonic patches is just beginning to be studied. Importantly, immunodeficient mouse strains including Il22ra1 ${ }^{-1-}$ and $\mathrm{Ragl}^{-1-}$ that succumb to $C$. rodentium infection have impaired innate and adaptive immune responses, respectively, and exhibit increased bacterial colonization of crypts and translocation relative to wild-type mice. ${ }^{27,28}$ This suggests that interleukin-22 (IL-22) signaling and some aspects of adaptive immunity are critical for protecting the crypt epithelium during infection with $\mathrm{A} / \mathrm{E}$ enteropathogens, although the basis for this is not understood.

\section{INNATE IMMUNE RESPONSE TO C. RODENTIUM}

Upon attachment of $C$. rodentium, epithelial cells are activated to release anti-microbial proteins, serum amyloid $\mathrm{A}$, and reactive oxygen species as a first line of defense to quarantine the pathogen to their apical surface. ${ }^{29-31}$ Subsequently, antigenpresenting cells, migratory monocytes, and other phagocytes use toll-like receptors and inflammasomes, consisting of intracellular NOD-like receptors, as well as signals provided by damaged epithelial cells to recognize and respond to C. rodentium infection. ${ }^{29,30,32-36} \mathrm{CX} 3 \mathrm{CR} 1^{+}$macrophages, $\mathrm{CCR}^{+}$macrophages, $\mathrm{CD} 11 \mathrm{~b}^{+} \mathrm{F} 4 / 80^{+} \mathrm{Gr} 1^{+}$inflammatory monocytes, and $\mathrm{CD} 11 \mathrm{~b}^{+} \mathrm{Gr}^{+}{ }^{+} \mathrm{Ly}_{6 \mathrm{G}}{ }^{\text {hi }}$ neutrophils are important phagocytes that are required for host defense against enteropathogenic infection. ${ }^{24,33-35,37,38}$ While phagocytes release proinflammatory cytokines and take up and lyse C. rodentium, dendritic cells (DCs) also migrate to the draining 
lymph nodes to prime and imprint circulating lymphocytes with chemokine receptors that direct them to the colonic lamina propria where they perform their effector functions. In particular, Notch2-dependent $\mathrm{CD} 11 \mathrm{~b}^{+} \mathrm{CD}_{103}{ }^{+}$DCs are an important source of the host-protective cytokine IL-23 in this infection model. ${ }^{35} \mathrm{IL}-23$ enhances the production of IL-22 from group 3 ILCs (ILC3s), neutrophils, and $\mathrm{CD}^{+} \mathrm{T}$ cells, particularly T-helper type 17 (Th17) and Th22 cells. ${ }^{39}$ IL-22 has emerged as a central protective cytokine in the C. rodentium infection model. ${ }^{40-42}$ IL-22 is an IL-10 family member cytokine that is reported to promote barrier defense at a range of mucosal surfaces and has an indispensible role in protection against infection with $C$. rodentium. ${ }^{43,44}$ A number of mechanisms have been proposed to explain how IL-22 mediates protection against enteropathogens, including enhanced bactericidal peptide expression, wound healing, mucin fucosylation, and complement production (see below). ${ }^{28,45}$ Other important proinflammatory cytokines produced by antigen-presenting cells in this infection include IL-1 $\beta$, IL-6, and IL-12. ${ }^{46-48}$ Collectively, these cytokines provide critical signals for the activation of effector functions from innate and adaptive lymphocytes during enteropathogenic infection.

\section{ADAPTIVE IMMUNE RESPONSE TO C. RODENTIUM}

Studies of the immune response to $C$. rodentium have shown that $\mathrm{CD} 4{ }^{+} \mathrm{T}$ cells and $\mathrm{B}$ cells are necessary for the clearance of this enteropathogen, but $\mathrm{CD} 8{ }^{+}$T cells are dispensable. ${ }^{49} \mathrm{CD} 28$ and $\mathrm{CD} 40 \mathrm{~L}$ costimulation and T-dependent immunoglobulin G (IgG) antibodies are required for the clearance of C. rodentium, but secreted IgA and IgM are not. ${ }^{50-52}$ Additional studies have demonstrated that the FcRn and Fcy receptors, complement component $\mathrm{C} 3$, and a broad antibody repertoire are protective during $C$. rodentium infection, suggesting that IgG is the predominant $\mathrm{T}$-dependent antibody isotype to promote intestinal barrier integrity, potentially via complement activation and increased phagocytosis. ${ }^{24,45,53-55}$ Collectively, these studies suggest that the $\mathrm{T}$ follicular helper (Tfh) cell subset is likely important in immunity to C. rodentium; however, the developmental relationship between Tfh cells and the other $\mathrm{CD} 4{ }^{+}$T-cell populations that predominate in this infection model have not been thoroughly examined and warrant further study ${ }^{52,56}$ For example, Th1, Th17, and Th22 cells are also thought to mediate host defense against $C$. rodentium via production of their signature cytokines interferon- $\gamma$ (IFN- $\gamma$ ), IL-17A, and IL-22, which enhance macrophage activation, attract neutrophils, and modulate gene expression in the epithelium, respectively. ${ }^{57} \mathrm{CD} 4{ }^{+} \mathrm{T}$ cells tend to initially produce IL-17A and IL-22 in response to $C$. rodentium infection, but as infection progresses, IFN- $\gamma$ production and epithelial damage increase, along with a reduction in the number of goblet cells. ${ }^{30,58,59}$ While it is not yet clear why this occurs, it appears that IFN- $\gamma$ signaling is beneficial to the host, as its absence is associated with delayed bacterial clearance and increased goblet cell numbers. ${ }^{47,58}$ Nevertheless, neutralization of IFN- $\gamma$ or IL-12 in the setting of IL-23 deficiency enhanced the survival of $I l 23 p 19^{-1-}$ mice, suggesting that the regulation of
Th17 to Th1 transition is a complex and incompletely characterized, yet important process in immunity to C. rodentium infection. ${ }^{37}$ As indicated previously, multiple studies have observed that $\mathrm{CD} 4{ }^{+} \mathrm{T}$ cells, ILC3s, and $\gamma \delta \mathrm{T}$ cells produce IL-17 and IL-22. ${ }^{40,59,60}$ While relative contributions of these populations to protection against $C$. rodentium infection have been debated, here we summarize recent findings and promote the concept of integrated type 3 immunity that requires the interplay of IL-22-producing ILC3s and CD4 ${ }^{+}$ $\mathrm{T}$ cells, ${ }^{32,60}$ which appear to have both redundant and nonredundant functions.

\section{SPATIOTEMPORAL CONTROL OF THE INTERPLAY BETWEEN IL-22-PRODUCING INNATE AND ADAPTIVE LYMPHOCYTES DURING C. RODENTIUM INFECTION}

ILC3s, Th17 cells, and Th22 cells share several phenotypic and developmental attributes. ${ }^{61,62}$ Importantly, ROR $\gamma \mathrm{t}$ and Ahr regulate the production of IL-22 from each cell type. ${ }^{61}$ Because neutralization of IL-22 before the peak of the CD4 ${ }^{+}$T-cell response was lethal, Zheng et al. ${ }^{43}$ speculated that IL-22producing $\mathrm{CD}^{+} \mathrm{T}$ cells were dispensable for immunity to C. rodentium infection and that $\mathrm{CD}_{11 \mathrm{c}^{+}}$DCs were the protective source of IL-22. ${ }^{43}$ Subsequently, it was demonstrated that IL-22-producing ROR $\gamma \mathrm{t}^{+} \mathrm{CD} 4{ }^{+}$lymphoid tissue inducer cells were an abundant source of IL-22 in the colonic intraepithelial layer of $R a g 1^{-1-}$ mice that lack adaptive lymphocytes; since these cells protected $I l 22^{-1-}$ mice from C. rodentium infection, whereas unpolarized $\mathrm{CD} 4^{+} \mathrm{T}$ cells did not, it was further assumed that $\mathrm{CD} 4^{+} \mathrm{T}$ cells might be dispensable as a source of IL-22 during infection. ${ }^{63}$ Additional ROR $\gamma \mathrm{t}$-dependent IL-22-producing ILCs were identified, including natural killer-like cells that express the natural cytotoxicity receptor (NCR) NKp46. ${ }^{64}$ These cells were similarly protective during $C$. rodentium infection in the absence of adaptive lymphocytes. ${ }^{64} \mathrm{IL}^{2} 2$-producing $\mathrm{NCR}^{+}$ and $\mathrm{NCR}^{-}$ILCs (the latter including $\mathrm{CD}^{+}$and $\mathrm{CD}^{-}$ lymphoid tissue inducer cells) were reclassified as ILC3s to avoid confusion and separate these cells from other ILCs that lack IL-22 production. ${ }^{61}$

Interestingly, whereas ILC3s are initially an abundant source of IL-22 following infection, CD4 ${ }^{+} \mathrm{T}$ cells soon become the dominant source of IL-22 as they migrate into the colonic lamina propria around day 5 of infection. ${ }^{40,59}$ Indeed, Basu et al. ${ }^{40}$ found that ILC3s fail to produce IL-22 in Il23p $19^{-1-}$ mice infected with a low-dose inoculum of $C$. rodentium, yet these mice survive and are protected via IL-23-independent production of IL- 22 by $\mathrm{CD} 4{ }^{+} \mathrm{T}$ cells. In contrast, IL- 6 was essential for production of IL-22 from $\mathrm{CD} 4{ }^{+} \mathrm{T}$ cells and host protection, consistent with the requirement for IL- 6 in the development of Th17 family effector cells. Interestingly, adoptive transfer studies demonstrated that Th 22 cells derived ex vivo were more protective against $C$. rodentium than conventional Th17 cells on a per cell basis following transfers into Il22 $2^{-1-}$ mice. ${ }^{40}$ Because ILC3s are the dominant source of IL-22 before day 5 , this suggests that ILC3s and $\mathrm{CD} 4{ }^{+} \mathrm{T}$ cells must both provide IL-22 to protect against C. rodentium 
infection-albeit at different tempos. Recently, using novel mouse strains to assess the relative requirement of IL-22 expression by ILC3s in the presence of T cells, it was found that IL-22 production by $\mathrm{NCR}^{+}$ILC3s is redundant in immunocompetent mice, where $\mathrm{CD}^{+} \mathrm{T}$ cells are present during C. rodentium infection. ${ }^{32,42,60,65}$ In view of the initial findings that implicate an important role for $\mathrm{NCR}^{-}$ILC3s early in infection, these observations imply that $\mathrm{NCR}^{-}$ILC3s might be more critical to early host protection before the development of IL-22-producing $\mathrm{CD} 44^{+} \mathrm{T}$ cells.

In a study that used an intimin-deficient strain of C. rodentium that cannot attach to the epithelium, it was demonstrated that the production of IL-22 by ILC $3 \mathrm{~s}$ is triggered by the presence of luminal $C$. rodentium, whereas the production of IL-17 by $\mathrm{CD} 4{ }^{+} \mathrm{T}$ cells requires epithelial attachment ${ }^{24}$ (Figure 1). This suggests that ILC3s are activated by luminal $C$. rodentium before the peak of bacterial colonization and that the cytokine milieu established by these cells might foster the induction and expansion of Th17 cells and induce IL-22 by CD $4 \mathrm{~T}$ cells in the subsequent phase of the infection. Indeed, it is possible that ILC3 cells recognize $C$. rodentium infection in the absence of a functional type III secretion system in a manner consistent with inflammasome activation in bone marrow-derived macrophages infected with the ATPase-deficient $\Delta \mathrm{EscN}$ mutant C. rodentium. ${ }^{36}$

Recently, a fate reporter mouse was generated to mark all cells that have transcribed IL-22 regardless of continued IL-22 production. ${ }^{59}$ Using this model, it was found that IL-22producing $\mathrm{CD}^{+}{ }^{+} \mathrm{T}$ cells expanded during infection and were an abundant source of IL-22 after day 5 of infection, consistent with previous results, ${ }^{12}$ whereas ILCs and $\gamma \delta$ T cells produced IL-22 earlier in infection but did not expand. ${ }^{59}$ In addition, IL-22-producing $\mathrm{CD} 4{ }^{+} \mathrm{T}$ cells exhibited greater plasticity as indicated by the frequency of cells coexpressing IFN- $\gamma$ or IL-17A relative to ILC3s, which primarily retained IL-22 production throughout the infection. ${ }^{59}$ Notably, $\mathrm{IL}^{-22} 2^{+}$fate reporter cells were clustered in the isolated lymphoid follicles on day 5 of $C$. rodentium infection, but were present in the lamina propria by day 25 of the infection. ${ }^{59}$ This suggests that ILC3s reside in isolated lymphoid follicles and are the predominant producers of IL-22 early in infection, whereas $\mathrm{CD} 4{ }^{+} \mathrm{T}$ cells are recruited to the lamina propria and dominate later. Interestingly, IL-22 expression by $\mathrm{CD}^{+}{ }^{+} \mathrm{T}$ cells was initially greater in the colonic lamina propria than in the mesenteric lymph nodes, ${ }^{66}$ suggesting that either the programming for IL-22 production by T cells is completed only after migration from the draining lymph nodes to the mucosa or that induction of T-cell priming might occur locally in the colonic mucosa. It is currently unclear to what extent the early ILC3 response conditions the induction and/or recruitment of $\mathrm{CD}^{+} \mathrm{T}$ cells, but there is clearly a spatiotemporal difference in the production of IL-22 by these innate and adaptive lymphoid populations that is consistent with a more static, rapid-response characteristic of ILC3s compared with $\mathrm{T}$ cells.

\section{IL-22-DEPENDENT REGULATION OF INTESTINAL COMMENSALS AND ENTERIC PATHOGENS}

IL-22 enhances the production of the bactericidal proteins lipocalin-2, S100A8, S100A9, RegIII $\beta$, and RegIII $\gamma$, and promotes barrier integrity via the upregulation of the tight junction protein claudin- $1 .^{67}$ Lipocalin-2 sequesters iron from bacterial siderophores. ${ }^{68}$ S100A8 and S100A9 heterodimerize to form calprotectin, which chelates manganese and zinc from a broad array of microorganisms, including C. rodentium. ${ }^{69}$ RegIII $\beta$ binds to lipid A and is bactericidal against Gramnegative bacteria but not Gram-positive bacteria. ${ }^{70}$ In contrast, RegIII $\gamma$ binds to peptidoglycan and is bactericidal against Gram-positive bacteria but not Gram-negative bacteria. ${ }^{71}$ Thus, not surprisingly, RegIII $\gamma^{-1-}$ mice clear infection with C. rodentium normally; however, these mice exhibit increased colonization with the Gram-positive mucosa-associated bacterium, Enterococcus faecalis. ${ }^{28}$ Interestingly, delivery of exogenous RegIII $\gamma$ to $I l 22^{-1-}$ mice infected with C. rodentium increased their survival. ${ }^{43}$ This suggests that the induction of RegIII $\gamma$ by IL-22 could protect mice from the systemic dissemination of Gram-positive microorganisms during C. rodentium infection-induced colitis. ${ }^{28}$ Thus, IL-22-dependent regulation of the microbiota appears to be multifaceted and can have indirect actions.

IL-22 is reported to regulate the composition of the microbiota during inflammation, but whether it does so under homeostatic conditions is unclear. ${ }^{28,72,73}$ Furthermore, IL-22 has disparate effects on Enterobacteriaceae. While IL-22 inhibits the growth of C. rodentium and commensal E. coli, it enhances the growth of Salmonella typhimurium. ${ }^{67}$ More specifically, IL-22 reciprocally modulates the growth of S. typhimurium and E. coli because E. coli is susceptible to the metal-scavenging anti-microbial proteins lipocalin and calprotectin, whereas S. typhimurium is not. ${ }^{67}$ Thus, these data suggest that the anti-microbial effects of IL-22 on C. rodentium infection could occur directly or reflect a perturbation to the microbiota that alters niche competition.

Colonization resistance, or the ability of individual bacterial species to impede the colonization of successive species, was initially recognized in the 1950s and is the premise for probiotic regimens and fecal microbiota transfer, which has become an accepted therapeutic approach for treating antibiotic-resistant Clostridium difficile infections. ${ }^{74}$ Indeed, colonization of neonatal mice with Lactobacillus rhamnosus and L. helveticus on day 7 of life was found to enhance the survival of neonates infected with C. rodentium 7 days later. ${ }^{75}$ It was found that the murine pathobiont segmented filamentous bacterium was present in the cecum and ileum of mice from Taconic and was associated with elevated IL-22 production and resistance to C. rodentium infection compared with mice from Jackson Laboratories (Bar Harbor, ME) that lacked segmented filamentous bacterium. ${ }^{76}$ Interestingly, monocolonization of Jackson mice with segmented filamentous bacterium was found to impede subsequent $C$. rodentium infection. In another study, gnotobiotic $\mathrm{C} 3 \mathrm{H} / \mathrm{HeJ}$ mice were colonized with the microbiota of NIH Swiss strain that is resistant to C. rodentium infection 
Early (d1-4)

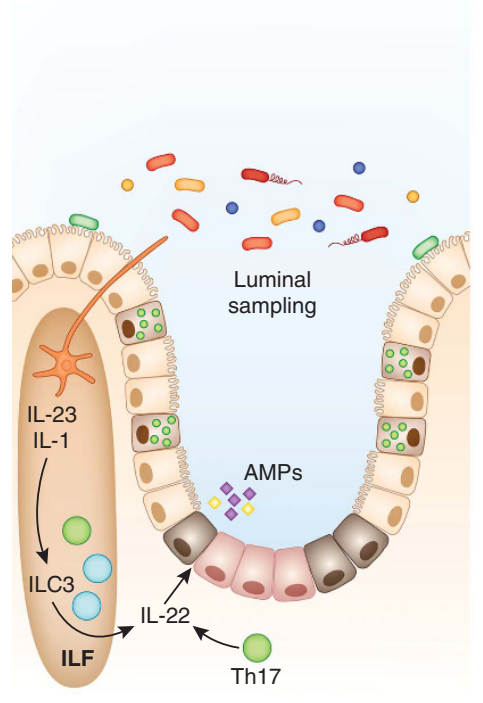

Peak (d5-12)

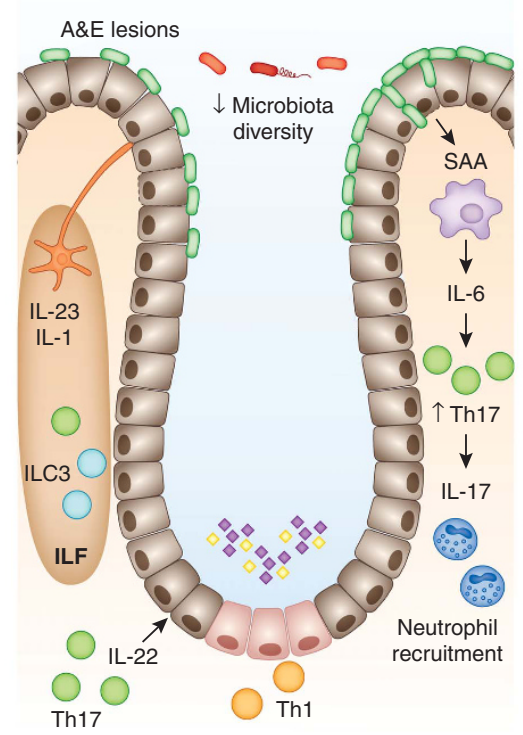

Late (d13-21)
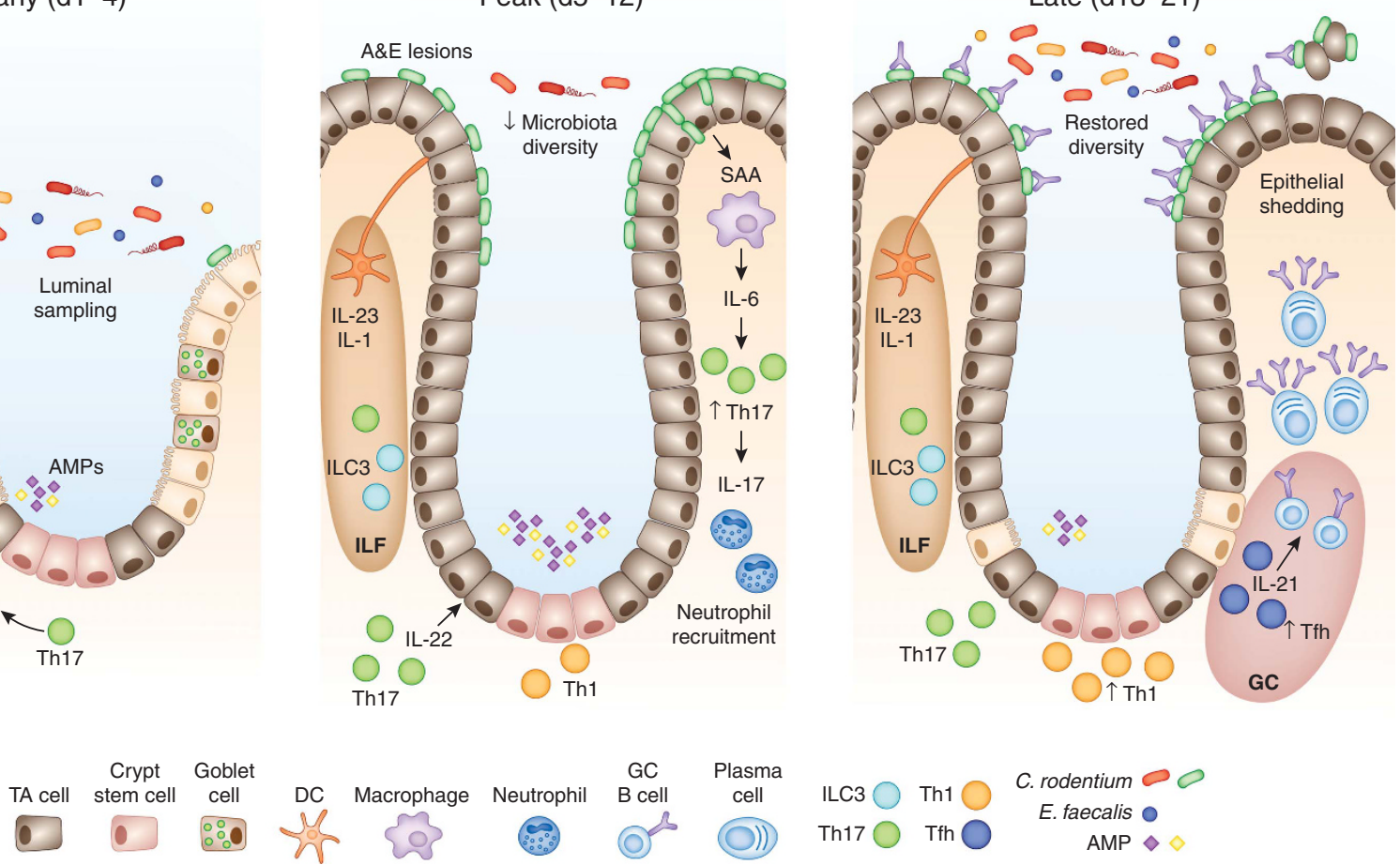

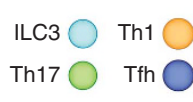

C. rodentium 0

E. faecalis 0

AMP $\diamond$

Figure 1 Enteropathogenic infection leads to dysbiosis of the microbiota and activation of type 3 immunity. $C$. rodentium attachment to the colonic epithelium is associated with the development and regulation of type 3 immune responses in the lamina propria at early (d1-4; left panel), middle (d5-12; middle panel), and late (d13-21; right panel) phases of the infection. Initially, dendritic cells (DCs) capture luminal $C$. rodentium (red), either directly or from microfold (M) cells overlying isolated lymphoid follicle (ILFs) and colonic patches that translocate bacteria from the lumen (not shown), and upregulate production of interleukin-1 (IL-1) and IL-23, which induce IL-22 expression from ILC3s. Attachment of host-adapted C. rodentium (green) to enterocytes (attaching and effacing, or A\&E, lesions) upregulates epithelial production of serum amyloid A (SAA), which induces the production of IL-6 from intestinal macrophages and promotes the expansion of T-helper type 17 (Th17) and Th22 cells. C. rodentium attachment induces epithelial hyperplasia and reduces the diversity of the microbiota, which is restored during the resolution of $C$. rodentium infection. Clearance of $C$. rodentium is associated with T-dependent antibody production, neutrophil recruitment, and an increase in interferon- $\gamma$ (IFN- $\gamma$ ) and IL-21 production.

and were designated as HeJ-NIH mice. ${ }^{77,78}$ Fecal microbiota transfer of the microbiota from these HeJ-NIH mice to $\mathrm{HeJ}$ mice transferred protection against $C$. rodentium to $\mathrm{HeJ}$ mice that otherwise succumb to infection; the enhanced survival in $\mathrm{HeJ}-\mathrm{NIH}$ mice relative to $\mathrm{HeJ}-\mathrm{HeJ}$ mice was correlated with elevated Il22 and RegIII $\beta$ mRNA. ${ }^{77}$ Moreover, the enhanced survival of HeJ-NIH mice during enteropathogenic infection was abrogated when IL-22 was neutralized, suggesting that some aspects of colonization resistance may be mediated via IL-22. ${ }^{77}$

Recently, IL-22 has been proposed to mediate protection against systemic dissemination of pathobionts via the fucosylation of mucins. For example, the $\alpha 1,2$-fucosyltransferase gene Fut2 is reduced in Il22 $\mathrm{ral}^{-1-}$ mice upon C. rodentium infection. ${ }^{28}$ While the Fut2 metabolite 2 fucosyllactose did not promote the clearance of $C$. rodentium, it did reduce the systemic dissemination of E. faecalis in Il22ral ${ }^{-1-}$ mice. In addition, similar studies with $C$. difficile infection-induced pathobiont dissemination further extend the anti-microbial properties of IL-22 to the upregulation of C3 mRNA expression and function in the liver and cecum. ${ }^{45}$ Thus, the activation of complement by IL-22 increases the versatility of IL-22dependent immune responses by further linking innate and adaptive lymphocytes with $\mathrm{CR} 1^{+}$phagocytes. Collectively, these studies suggest multiple levels at which IL-22 integrates immunity to enteric infection, including: modification of mucin fucosylation, anti-microbial peptide production, composition of the intestinal microbiota, and complement activity (Figure 2). Further studies will be required to unravel the hierarchy of these effects in modulating IL-22-dependent barrier defense.

\section{IL-21-DEPENDENT REGULATION OF ENTERIC PATHOGENS}

Because IL-22 can be induced by IL-21 and $I I 21^{-1-}$ mice had delayed clearance of $C$. rodentium, ${ }^{57}$ we became interested in the similarities and differences between IL-21 and IL-22 in this infection model. ${ }^{40,79}$ Whereas both innate and adaptive immune cells produce IL-22, CD4 ${ }^{+} \mathrm{T}$ cells are thought to be the primary source of IL-21. ${ }^{80} \mathrm{IL}-21$ is a pleiotropic cytokine that has been reported to enhance the production of the IL-23R and maintain IL-17 production from $\mathrm{CD}^{+}{ }^{+} \mathrm{T}$ cells while inhibiting the expression of Foxp $3{ }^{80,81}$ While IL-21 increases the expression of IL-22 from CD4 ${ }^{+} \mathrm{T}$ cells cultured in vitro, direct ex vivo analysis demonstrated that $\mathrm{CD} 4^{+} \mathrm{T}$ cells from mice deficient for both IL-2 and IL-21 expressed increased IL-22 relative to $\mathrm{CD} 4^{+} \mathrm{T}$ cells from mice deficient for IL-2 alone. This suggests that the interaction between IL-21 and IL-22 is more complex than is evident from in vitro studies. ${ }^{82}$ 

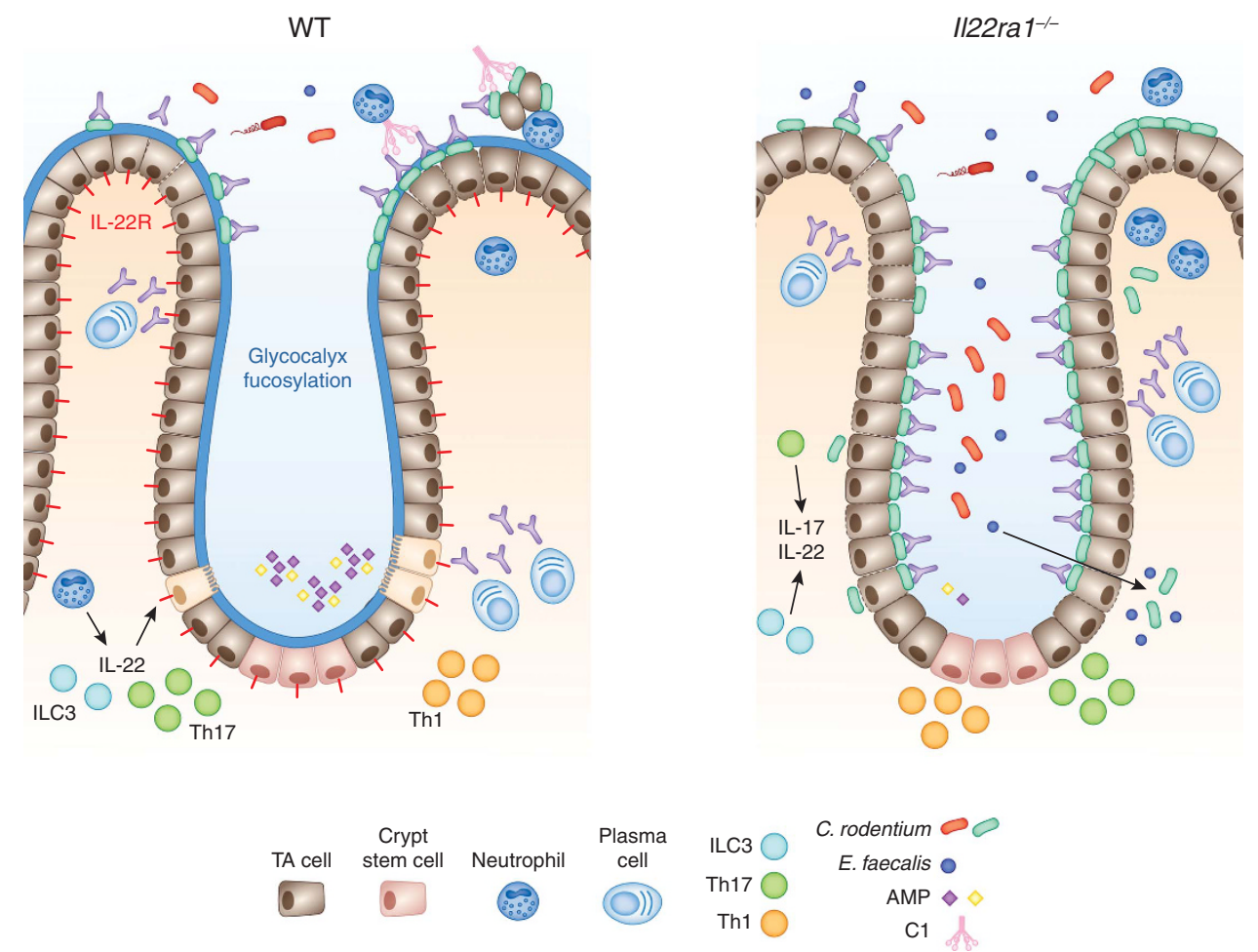

Figure 2 Interleukin-22 (IL-22)-dependent actions that contribute to host defense during $C$. rodentium infection. During type 3 immune responses to C. rodentium infection, IL-22 promotes host protection by upregulating epithelial wound repair and anti-microbial peptide production. In particular, IL-22 enhances the mRNA expression of the gap junction protein claudin- 1 and the anti-microbial peptides Reglll $\beta$, Reglll $\gamma, \mathrm{S} 100 \mathrm{~A} 8$, S100A9, and lipocalin-2 in the colon. ${ }^{67}$ S100A8 and S100A9 heterodimerize to form calprotectin, which sequesters zinc and manganese from pathogens and commensals. Although not directly bactericidal against $C$. rodentium, Reglll $\gamma$ likely enhances survival of WT mice by preventing the dissemination of mucosaassociated Gram-positive pathobionts, such as E. faecalis, which occurs in $/ 122 \mathrm{ra1} 1^{-1}-$ mice. ${ }^{28} \mathrm{IL}-22$ further regulates the fucosylation of mucins forming the gylcocalyx and production of the $\mathrm{C} 3$ component of complement that contribute to alteration of the microbiota composition and prevent systemic dissemination of pathobionts during enteric infection. ${ }^{28,45}$

Clearly, IL-21 has an important role in protecting mucosal surfaces from infection as humans with IL-21 and IL-21R deficiency exhibit increased susceptibility to parasitic, viral, and bacterial infections, ${ }^{83-85}$ but how it contributes, and whether or not it has a role in modulating IL-22 responses, is yet to be fully characterized.

To further probe the role of IL-21 in host defense in the intestine, Basu et al. ${ }^{40}$ explored the susceptibility of $I l 21^{-1-}$ mice to infection with C. rodentium. Unlike Il22 ${ }^{-1-}$ mice, $I l 21^{-1-}$ mice did not succumb to the infection; however, as indicated previously, $I l 21^{-1-}$ mice exhibit substantially delayed clearance of this enteropathogen. ${ }^{40}$ Because of the time lag associated with the activation and expansion of pathogen-specific $\mathrm{CD} 4{ }^{+} \mathrm{T}$ cells during infection, it is likely that the levels of IL-21 immediately following infection are low and that IL-21 production does not peak until the height of the $\mathrm{CD}^{+}{ }^{+} \mathrm{T}$-cell response. Thus, the kinetics of IL-21 production may have important implications for the likely targets of IL-21 signaling in this model and highlight key differences between the function of IL-21 and IL-22 in mucosal immunity. A key feature that distinguishes IL-21 and IL-22 signaling is that the expression of IL-22RA1 is restricted to non-hematopoietic cells while the IL-21R is expressed by multiple lineages, including lymphocytes and non-hematopoietic cells. Thus, the diversity of cells expressing the IL-21R suggests multiple potential routes by which IL-21 could contribute the clearance of $C$. rodentium.

The delayed clearance of $C$. rodentium in $I l 21^{-1-}$ mice is consistent with intact innate immunity but an impaired adaptive immune response, although it is unknown whether this reflects defects in T-cell responses, B-cell responses, or both. Because of the importance of IL-21 in antibody production, it is likely that $C$. rodentium-specific antibodies are diminished in $I l 21^{-1-}$ mice relative to wild-type mice. ${ }^{86}$ IL-21 is highly produced by conventional CXCR $5^{+} \mathrm{PD}-1^{+} \mathrm{Tfh}$ but inhibits the expression of Foxp 3 by $\mathrm{CD} 4^{+} \mathrm{T}$ cells, raising the possibility that IL-21 deficiency alters the balance between Tfh cells, Foxp ${ }^{+}$Treg cells, and T follicular regulatory cells so as to impair the $C$. rodentium-specific IgG response during infection $^{81,87,88}$ (Figure 3). Whether IL-21 regulates the balance of these cells by modulating their survival, migration, interconversion, or death is currently unknown, but recent data indicate that IL-21 signaling may inhibit the proliferation of $\mathrm{T}$ follicular regulatory cells. ${ }^{89,90}$ Nevertheless, it would appear that sufficient antibody production remains to eradicate the infection, as bacterial clearance is delayed, but not lost, in $I l 21^{-1-}$ mice. ${ }^{12}$ It is unknown whether IL-21 deficiency 

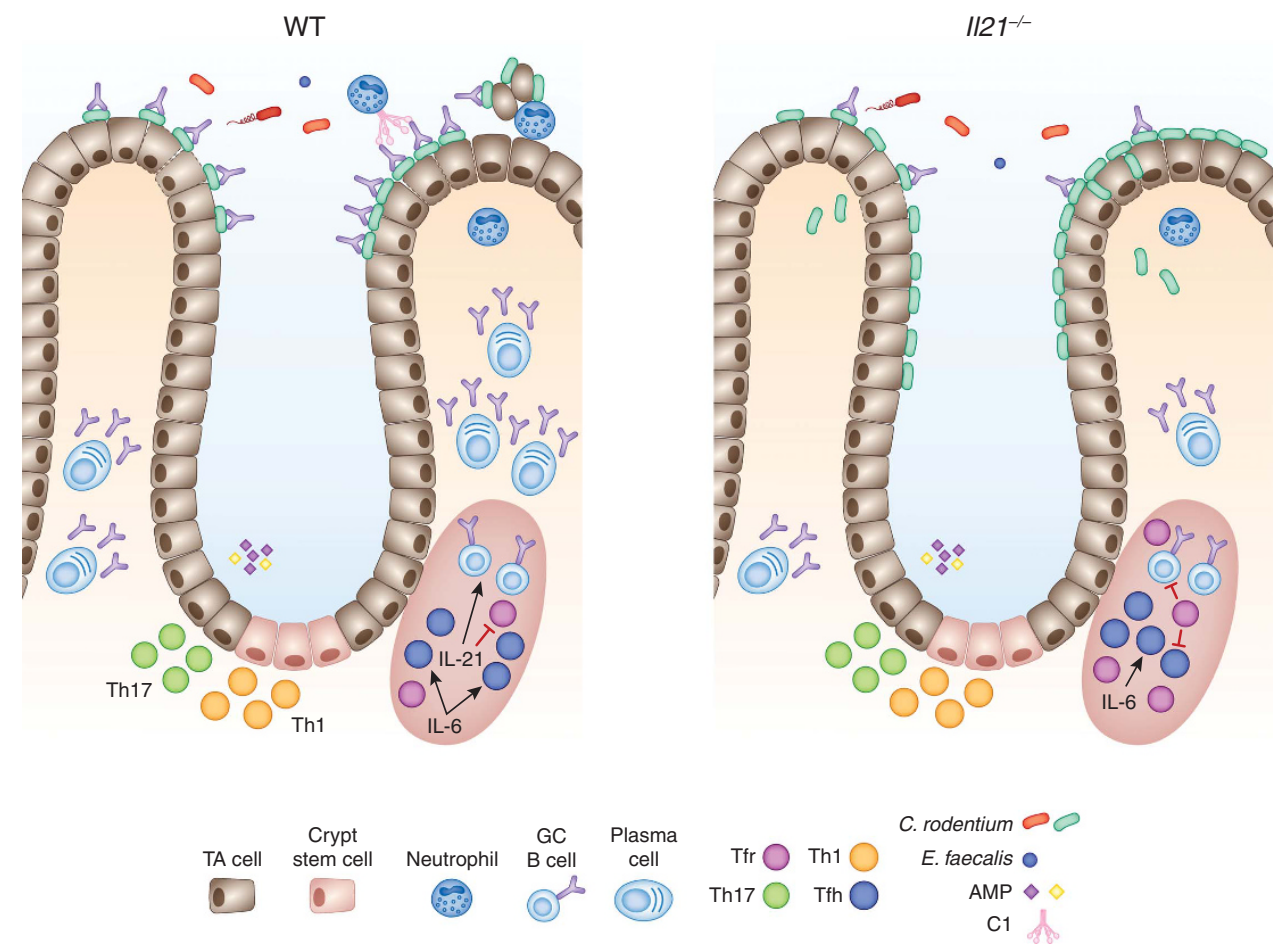

Figure 3 Interleukin-21 (IL-21) enhances humoral immunity to enteropathogens. During type 3 immune responses IL-6 induces IL-21 production by $\mathrm{CD}^{+}{ }^{+} \mathrm{T}$ cells and promotes the clearance of $C$. rodentium infection. IL-21 is highly produced by both T follicular helper (Tfh) cells and T-helper type 17 (Th17) cells, inhibits Foxp3 expression, and alters the Tfh to T follicular regulatory (Tfr) balance via undefined mechanisms. ${ }^{87,88} \mathrm{IL}-21$ enhances plasma cell differentiation and immunoglobulin $\mathrm{G}(\mathrm{IgG})$ production. ${ }^{86}$

impairs Th17 and Th22 responses during infection, although IL-21 can enhance IL-6-driven Th17 development in vitro. Each of these issues will require further study.

To date, most experimental models of infection or immunization designed to probe the function of IL-21 in mice have focused on the systemic immune system rather than specific mucosal sites; however, IL-21 was shown to enhance the rate of clearance of the gastrointestinal bacterial and parasitic pathogens Helicobacter pylori and Toxoplasma gondii, respectively. ${ }^{86,91-95}$ Interestingly, administration of IL-21 to SIVinfected rhesus macaques reduces the systemic dissemination of LPS into circulation, suggesting that IL-21 prevents the translocation of Gram-negative bacteria from the intestine. ${ }^{96}$ These data suggest that the increased production of IL-21 observed upon perturbation to the intestinal microbiota, such as in patients with inflammatory bowel disease and mice treated with dextran sulfate sodium, might reflect a compensatory mechanism to restore homeostasis in the intestine; however, whether IL-21 regulates the composition of the intestinal microbiota has not been directly examined. ${ }^{97-99}$

\section{FUTURE PROSPECTS FOR TRACKING TYPE 3 IMMUNITY IN THE C. RODENTIUM INFECTION MODEL}

A shortcoming of the $C$. rodentium infection model is the lack of antigen-specific tools with which to track the fate and function of the pathogen-specific $\mathrm{CD} 4^{+} \mathrm{T}$ cells and $\mathrm{B}$ cells during infection. To our knowledge, the only reagents that are currently available to address this issue include transgenic strains of $C$. rodentium that express OVA or GFP, OT-II T-cell receptor transgenic mice, OVA tetramers, and peptide-major histocompatibility complex tetramers that contain immunodominant epitopes from OVA or GFP. ${ }^{56,78,100,101}$ In fact, the only study to examine the antigen-specific $\mathrm{CD} 4^{+}$T-cell response to $C$. rodentium infection used the OVA-expressing strain to demonstrate OVA-specific Th1 cells enhance IgG1 antibodies to $C$. rodentium. ${ }^{56}$ Adopting this strategy, or a similar one, to study antigen-specific $\mathrm{T}$-cell responses during C. rodentium infection would greatly improve our understanding of the $C$. rodentium-specific $\mathrm{CD} 4{ }^{+} \mathrm{T}$-cell subsets that develop during enteropathogenic infection and their overlapping and distinct functions relative to ILC3s. First, this approach would provide an opportunity to delineate the functions of C. rodentium-specific $\mathrm{CD}^{+} \mathrm{T}$ cells in the presence of an intact ILC3 compartment, as ILC3 cells lack expression of a T-cell receptor. Interestingly, because CCR6 ${ }^{+}$ Tbet $^{-} \mathrm{ROR} \gamma \mathrm{t}^{+}$ILC populations are reported to express major histocompatibility complex class II and function as antigenpresenting cells, ${ }^{102}$ this may also offer a means to better examine the interaction between ILC3s and $\mathrm{CD} 4{ }^{+} \mathrm{T}$ cells during enteropathogenic infection. Second, Tfh cells and T follicular regulatory cells have been widely studied in secondary lymphoid organs and the Peyer's patch of the small intestine, but have not been examined in the colon where the largest density of commensal antigens are located. ${ }^{92,95,103}$ Precisely 
how IgG affinity maturation occurs in this environment during enteropathogenic infection is not well understood, considering that many intestinal phagocytes are tolerant of luminal antigens in the steady state. Third, the development of transgenic antigen-specific model systems with which to study C. rodentium-specific adaptive responses would also enhance our understanding of both early immunological priming events and immunological memory associated with type 3 immunity following $\mathrm{A} / \mathrm{E}$ enteropathogen infection in the large intestine. ${ }^{56,104-106}$ Finally, without better methods for tracking clonal populations of $\mathrm{CD}^{+} \mathrm{T}$ cells and $\mathrm{B}$ cells during C. rodentium infection, it is difficult to understand how and where antigen-specific Th17 cells transition into Th1 cells during infection and the roles played by each in host defense.

Ideally, the utilization of $C$. rodentium strains that express model antigens should eventually be supplemented with additional reagents to track pathogen-specific lymphocytes based on the endogenous antigens of this bacterium. Because studies using intimin-deficient strains of $C$. rodentium in germfree mice revealed that LEE-encoded virulence factors are dominant antigens for this pathogen, peptide-major histocompatibility complex tetramers using epitopes from these virulence factors may be ideal. ${ }^{24}$ Interestingly, immunization of formalin-fixed $C$. rodentium promoted hyperplasia of the epithelium, IFN- $\gamma$ production, and the proliferation of $\mathrm{CD} 4^{+}$ $\mathrm{T}$ cells, whereas immunization of formalin-fixed $\Delta e a e$ C. rodentium that lacks intimin did not, indicating that intimin is required to induce $\mathrm{CD} 4^{+} \mathrm{T}$-cell proliferation and activation. ${ }^{107,108}$ Currently, it is unknown whether the intimininduced proliferation of $\mathrm{CD} 4{ }^{+} \mathrm{T}$ cells occurs via the interaction of intimin with integrins, or whether an immunodominant peptide within the intimin protein induces the proliferation of $\mathrm{CD} 4{ }^{+} \mathrm{T}$ cells; however, these studies occurred before the characterization of Th17 cells, which were recently shown to be activated by intimin-expressing C. rodentium. ${ }^{30}$ Thus, additional analyses of intimin-specific effects on $\mathrm{CD} 4^{+} \mathrm{T}$-cell subsets are warranted..$^{24,108,109} \mathrm{We}$ anticipate that these reagents will improve the characterization type 3 immune responses in the intestine and facilitate the generation of improved vaccines to $\mathrm{A} / \mathrm{E}$ enteropathogens.

\section{CONCLUSION}

C. rodentium has become a widely studied model of $\mathrm{A} / \mathrm{E}$ enteropathogenic infection and represents an important infectious model for understanding the dynamics of type 3 immunity in host defense. In this review, we have overviewed our current understanding of the dynamics of the innate and adaptive response to infection with $C$. rodentium with particular emphasis on our current understanding of the sources of IL-22 and IL-21 and their targets of action. Clearly, much remains to be explored. We envision that future studies using C. rodentium as a model enteric pathogen will increase our understanding of the interactions between innate and adaptive lymphocytes and could be of potential benefit for mucosal vaccinations designed to enhance the quality of Th17 and Tfh cell responses.

\section{ACKNOWLEDGMENTS}

This work was supported by grants from NIH (to D.J.S. and C.T.W.) and the Crohn's and Colitis Foundation of America (to C.T.W.).

\section{AUTHOR CONTRIBUTIONS}

D.J.S. C.L.Z. and C.T.W. cowrote this article.

\section{DISCLOSURE}

The authors declared no conflict of interest.

c 2017 Society for Mucosal Immunology

\section{REFERENCES}

1. Liu, L. et al. Global, regional, and national causes of child mortality: an updated systematic analysis for 2010 with time trends since 2000. Lancet 379, 2151-2161 (2012).

2. Kotloff, K.L. et al. Burden and etiology of diarrheal disease in infants and young children in developing countries (the Global Enteric Multicenter Study, GEMS): a prospective, case-control study. Lancet 382, 209-222 (2013).

3. World Health Organization (WHO) World Health Statistics, 2015. pp 1-164. Available at http://www.who.int/gho/publications/world health_statistics/2015/en/. Accessed 14 September 2015.

4. Croxen, M.A. et al. Recent advances in understanding enteric pathogenic Escherichia coli. Clin. Microbiol. Rev. 26, 822-880 (2013).

5. Nataro, J.P. \& Kaper, J.B. Diarrheagenic Escherichia coli. Clin Microbiol Rev 11, 142-201 (1997).

6. Centers for Disease Control and Prevention (CDC). Diarrheagenic E. coli (US Department of Health and Human Services, Center for Disease Control and Prevention, Atlanta, GA). Available at http://www.cdc.gov/ ecoli/diarrheagenic-ecoli.html. Accessed 8 July 2016.

7. Centers for Disease Control and Prevention (CDC). Foodborne Outbreak Online Database. (US Department of Health and Human Services, Center for Disease Control and Prevention, Atlanta, GA). http://wwwn.cdc.gov/ foodborneoutbreaks/. Accessed 27 April 2016.

8. Mundy, R. et al. Citrobacter rodentium of mice and man. Cell. Microbiol. 7, 1697-1706 (2005).

9. Deng, W. et al. Locus of enterocyte effacement from Citrobacter rodentium: sequence analysis and evidence for horizontal transfer among attaching and effacing pathogens. Infect. Immun. 69, 6323-6335 (2001).

10. McDaniel, T.K. \& Kaper, J.B. A cloned pathogenicity island from enteropathogenic Escherichia coli confers the attaching and effacing phenotype on E. coli K-12. Mol. Microbiol. 23, 399-407 (1996).

11. Lewis, S. et al. Enterohemorrhagic Escherichia coli colonization of human colonic epithelium in vitro and ex vivo. Infect. Immun. 83, 942-949 (2015).

12. Schüller, S. et al. The ex vivo response of human intestinal mucosa to enteropathogenic Escherichia coli infection. Cell. Microbiol. 11, 521-530 (2009).

13. In, J. et al. Enterohemorrhagic Escherichia coli reduces mucus and intermicrovillar bridges in human stem cell-derived colonoids. Cell. Mol. Gastroenterol. Hepatol. 2, 48-62.e3 (2016).

14. Collins, J.W. et al. Citrobacter rodentium: infection, inflammation and the microbiota. Nat. Immunol. 12, 612-623 (2014).

15. Wiles, S. et al. Modelling infectious disease-time to think outside the box?. Nat. Immunol. 4, 307-312 (2006).

16. Mallick, E.M. et al. A novel murine infection model for shiga toxinproducing Escherichia coli. J. Clin. Invest. 122, 4012-4024 (2012).

17. Deng, W. et al. Dissecting virulence: systematic and functional analyses of a pathogenicity island. Proc. Natl Acad. Sci. USA 101, 3597-3602 (2004).

18. Ghaem-Maghami, M. et al. Intimin-specific immune responses prevent bacterial colonization by the attaching-effacing pathogen Citrobacter rodentium. Infect. Immun. 69, 5597-5605 (2001).

19. McNeilly, T.N. et al. Immunization of cattle with a combination of purified intimin-531, EspA and Tir significantly reduces shedding of Escherichia coli O157:H7 following oral challenge. Vaccine 28, 1422-1428 (2010). 
20. Yu, S. et al. Identification of a novel linear epitope on EspA from enterohemorrhagic $E$. coli using a neutralizing and protective monoclonal antibody. Clin. Immunol. 138, 77-84 (2011).

21. Wiles, S. et al. Emergence of a 'hyperinfectious' bacterial state after passage of Citrobacter rodentium through the host gastrointestinal tract. Cell. Microbiol. 7, 1163-1172 (2005).

22. Kamada, N. et al. Regulated virulence controls the ability of a pathogen to compete with the gut microbiota. Science 336, 1325-1329 (2012).

23. Deng, W. et al. Regulation of type III secretion hierarchy of translocators and effectors in attaching and effacing bacterial pathogens. Infect. Immun. 73, 2135-2146 (2005).

24. Kamada, N. et al. Humoral immunity in the gut selectively targets phenotypically virulent attaching-and-effacing bacteria for intraluminal elimination. Cell Host Microbe 17, 617-627 (2015).

25. Lupp, C. et al. Host-mediated inflammation disrupts the intestinal microbiota and promotes the overgrowth of Enterobacteriaceae. Cell Host Microbe 2, 119-129 (2007).

26. Lopez, C.A. et al. Virulence factors enhance Citrobacter rodentium expansion through aerobic respiration. Science 353, 1249-1253 (2016).

27. Vallance, B.A. et al. Mice lacking T and B lymphocytes develop transient colitis and crypt hyperplasia yet suffer impaired bacterial clearance during Citrobacter rodentium infection. Infect. Immun. 70, 2070-2081 (2002).

28. Pham, T.A.N. et al. Epithelial IL-22RA1-mediated fucosylation promotes intestinal colonization resistance to an opportunistic pathogen. Cell Host Microbe 16, 504-516 (2014).

29. Sano, T. et al. An IL-23R/IL-22 circuit regulates epithelial serum amyloid $A$ to promote local effector Th17 responses. Cell 163, 381-393 (2015).

30. Atarashi, K. et al. Th17 cell induction by adhesion of microbes to intestinal epithelial cells. Cell 163, 367-380 (2015).

31. Pedicord, V.A. \& Mucida, D. "A sledgehammer breaks glass but forges steel": bacteria adhesion shapes gut immunity. Cell 163, 273-274 (2015).

32. Song, C. et al. Unique and redundant functions of $\mathrm{NKp} 46^{+} \mathrm{ILC} 3 \mathrm{~s}$ in models of intestinal inflammation. J. Exp. Med. 212, 1869-1882 (2015).

33. Manta, C. et al. $\mathrm{CX}_{3} \mathrm{CR} 1^{+}$macrophages support IL-22 production by innate lymphoid cells during infection with Citrobacter rodentium. Mucosal Immunol. 6, 177-188 (2013).

34. Seo, S.-U. et al. Intestinal macrophages arising from CCR2 ${ }^{+}$monocytes control pathogen infection by activating innate lymphoid cells. Nat. Commun. 6, 1-12 (2015).

35. Satpathy, A.T. et al. Notch2-dependent classical dendritic cells orchestrate intestinal immunity to attaching-and-effacing bacterial pathogens. Nat. Immunol. 14, 937-948 (2013).

36. Liu, Z. et al. Role of inflammasomes in host defense against Citrobacter rodentium infection. J. Biol. Chem. 287, 16955-16964 (2012).

37. Aychek, T. et al. IL-23-mediated mononuclear phagocyte crosstalk protects mice from Citrobacter rodentium-induced colon immunopathology. Nat. Commun. 6, 1-10 (2015).

38. Kim, Y.-G. et al. The Nod2 sensor promotes intestinal pathogen eradication via the chemokine CCL2-dependent recruitment of inflammatory monocytes. Immunity 34, 769-780 (2011).

39. Zindl, C.L. et al. IL-22-producing neutrophils contribute to antimicrobial defense and restitution of colonic epithelial integrity during colitis. Proc. Natl Acad. Sci. USA 110, 12768-12773 (2013).

40. Basu, R. et al. Th22 cells are an important source of IL-22 for host protection against enteropathogenic bacteria. Immunity 37, 1061-1065 (2012).

41. Klose, C.S.N. \& Artis, D. Innate lymphoid cells as regulators of immunity, inflammation and tissue homeostasis. Nat. Immunol. 17, 765-774 (2016).

42. Bando, J.K. \& Colonna, M. Innate lymphoid cell function in the context of adaptive immunity. Nat. Immunol. 17, 783-789 (2016).

43. Zheng, Y. et al. Interleukin-22 mediates early host defense against attaching and effacing bacterial pathogens. Nat. Med. 14, 282-289 (2008).

44. Ouyang, W. et al. Regulation and functions of the IL-10 family of cytokines in inflammation and disease. Annu. Rev. Immunol. 29, 71-109 (2011).

45. Hasegawa, M. et al. Interleukin-22 regulates the complement system to promote resistance against pathobionts after pathogen-induced intestinal damage. Immunity 41, 620-632 (2014).

46. Dann, S.M. et al. IL-6-dependent mucosal protection prevents establishment of a microbial niche for attaching/effacing lesion-forming enteric bacterial pathogens. J. Immunol. 180, 6816-6826 (2008).
47. Simmons, C.P. et al. Impaired resistance and enhanced impaired pathology during infection with a noninvasive, attaching-effacing enteric bacterial pathogen, Citrobacter rodentium, in mice lacking IL-12 or IFN- $\gamma$. J. Immunol. 168, 1804-1812 (2002).

48. Lebeis, S. L. etal. Interleukin-1 receptor signaling protects mice from lethal intestinal damage caused by the attaching and effacing pathogen Citrobacter rodentium. Infect. Immun. 77, 604-614 (2009).

49. Simmons, C.P. et al. Central role for B lymphocytes and CD4 ${ }^{+}$T cells in immunity to infection by the attaching and effacing pathogen Citrobacter rodentium. Infect. Immun. 71, 5077-5086 (2003).

50. Bry, L. et al. B. CD4 ${ }^{+} \mathrm{T}$ cell effector functions and costimulatory requirements essential for surviving mucosal infection with Citrobacter rodentium. Infect. Immun. 74, 673-681 (2005).

51. Maaser, C. et al. Clearance of Citrobacter rodentium requires B cells but not secretory immunoglobulin A (IgA) or IgM antibodies. Infect. Immun. 72, 3315-3324 (2004).

52. Linterman, M.A. et al. CD28 expression is required after $T$ cell priming for helper $\mathrm{T}$ cell responses and protective immunity to infection. elife 3, 1-21 (2014).

53. Yoshida, M. et al. Neonatal Fc receptor for IgG regulates mucosal immune responses to luminal bacteria. J. Clin. Invest. 116, 2142-2151 (2006).

54. Masuda, A. et al. Fc receptor regulation of Citrobacter rodentium infection. Infect. Immun. 76, 1728-1737 (2008).

55. Belzer, C. et al. The role of specific lgG and complement in combating a primary mucosal infection of the gut epithelium. Eur. J. Microbiol. Immunol. 1, 311-318 (2011).

56. Shiomi, H. etal. Gamma interferon produced by antigen-specific $C D 4^{+} \mathrm{T}$ cells regulates the mucosal immune responses to Citrobacter rodentium infection. Infect. Immun. 78, 2653-2666 (2010).

57. Murphy, K. \& Weaver, C. Janeway's Immunobiology. Garland Science 2016.

58. Chan, J.M. et al. CD4 ${ }^{+} \mathrm{T}$ cells drive goblet cell depletion during Citrobacter rodentium infection. Infect. Immun. 81, 4649-4658 (2013).

59. Ahlfors, H. et al. IL-22 fate reporter reveals origin and control of IL-22 production in homeostasis and infection. J. Immunol. 193, 4602-4613 (2014).

60. Rankin, L.C. et al. Complementarity and redundancy of IL-22-producing innate lymphoid cells. Nat. Immunol. 17, 179-186 (2016).

61. Spits, H. et al. Innate lymphoid -a proposal for uniform nomenclature. Nat. Rev. Microbiol. 13, 145-149 (2013).

62. Artis, D. \& Spits, H. The biology of innate lymphoid cells. Nature $\mathbf{5 1 7}$, 293-301 (2015).

63. Sonnenberg, G.F. et al. CD4+ lymphoid tissue-inducer cells promote innate immunity in the gut. Immunity 34, 122-134 (2011).

64. Satoh-Takayama, N. et al. Microbial flora drives interleukin 22 production in intestinal $\mathrm{NKp} 46^{+}$cells that provide innate mucosal immune defense. Immunity 29, 958-970 (2008).

65. Vivier, E. et al. The evolution of innate lymphoid cells. Nat. Immunol. 17, 790-794 (2016).

66. Song, X. et al. IL-17RE is the functional receptor for IL-17C and mediates mucosal immunity to infection with intestinal pathogens. Nat. Immunol. 12, 1151-1158 (2011).

67. Behnsen, J. et al. The cytokine IL-22 promotes pathogen colonization by suppressing related commensal bacteria. Immunity 40, 262-273 (2014).

68. Flo, T.H. et al. Lipocalin 2 mediates an innate immune response to bacterial infection by sequestrating iron. Nature 432, 917-921 (2004).

69. Mukherjee, S. et al. Antibacterial membrane attack by a pore-forming intestinal C-type lectin. Nature 505, 103-107 (2014).

70. Miki, T. et al. The bactericidal activity of the C-type lectin Reglll $\beta$ against Gram-negative bacteria involves binding to lipid A. J. Biol. Chem. 287, 34844-34855 (2012).

71. Cash, H.L. et al. Symbiotic bacteria direct expression of an intestinal bactericidal lectin. Science 313, 1126-1130 (2006).

72. Zenewicz, L.A. et al. IL-22 deficiency alters colonic microbiota to be transmissible and colitogenic. J. Immunol. 190, 5306-5312 (2013).

73. Sonnenberg, G.F. et al. Innate lymphoid cells promote anatomical containment of lymphoid-resident commensal bacteria. Science 336, 1321-1325 (2012).

74. Borody, T.J. \& Khoruts, A. Fecal microbiota transplantation and emerging applications. Nat. Rev. Gastroenterol. Hepatol. 9, 88-97 (2011). 
75. Gareau, M.G. et al. Probiotics prevent death caused by Citrobacter rodentium infection in neonatal mice. J. Infect. Dis. 201, 81-91 (2010).

76. Ivanov, I.I. et al. Induction of intestinal Th17 cells by segmented filamentous bacteria. Cell 139, 485-498 (2009).

77. Willing, B.P. et al. Altering host resistance to infections through microbial transplantation. PLOS ONE 6, e26988 (2011).

78. Vallance, B.A. et al. Host susceptibility to the attaching and effacing bacterial pathogen Citrobacter rodentium. Infect. Immun. 71, 3443-3453 (2003).

79. Yeste, A. et al. IL-21 induces IL-22 production in CD4 ${ }^{+}$T cells. Nat. Commun. 5, 3753 (2014).

80. Spolski, R. \& Leonard, W.J. Interleukin-21: a double-edged sword with therapeutic potential. Nat. Immunol. 13, 379-395 (2014).

81. Nurieva, R. et al. Essential autocrine regulation by IL-21 in the generation of inflammatory T cells. Nature 448, 480-483 (2007).

82. Vogelzang, A. et al. IL-21 contributes to fatal inflammatory disease in the absence of Foxp3 ${ }^{+}$Tregulatory cells. J. Immunol. 192, 1404-1414 (2014).

83. Stepensky, P. et al. Extending the clinical and immunological phenotype of human interleukin-21 receptor deficiency. Haematologica 100, e72-e76 (2015).

84. Kotlarz, D. et al. Loss-of-function mutations in the IL-21 receptor gene cause a primary immunodeficiency syndrome. J. Exp. Med. 210, 433-443 (2013).

85. Salzer, E. et al. Early-onset inflammatory bowel disease and common variable immunodeficiency-like disease caused by IL-21 deficiency. J. Allergy. Clin. Immunol. 133, 1651-1659 (2014).

86. Ozaki, K. et al. A critical role for IL-21 in regulating immunoglobulin production. Science 298, 1630-1634 (2002).

87. Crotty, S. Follicular helper CD4 T cells (TFH). Annu. Rev. Immunol. 29, 621-663 (2011).

88. Ding, Y. et al. Interleukin-21 promotes germinal center reaction by skewing the follicular regulatory $T$ cell to follicular helper $T$ cell balance in autoimmune BXD2 mice. Arthritis Rheum. 66, 2601-2612 (2014).

89. Sage, P.T. et al. Suppression by TFR cells leads to durable and selective inhibition of B cell effector function. Nat. Commun. 17, 1-13 (2016).

90. Jandl, C. et al. IL-21 restricts $T$ follicular regulatory $T$ cell proliferation through Bcl-6 mediated inhibition of responsiveness to IL-2. Nat. Commun. 8, 1436-1446 (2017).

91. Yi, J.S. et al. A vital role for interleukin-21 in the control of a chronic viral infection. Science 324, 1572-1576 (2009).

92. Eto, D. et al. IL-21 and IL-6 are critical for different aspects of B cell immunity and redundantly induce optimal follicular helper CD4 T cell (Tfh) differentiation. PLOS ONE 6, e17739 (2011).

93. Lüthje, K. etal. The development and fate of follicular helper Tcells defined by an IL-21 reporter mouse. Nat. Immunol. 13, 491-498 (2012).
94. Carbo, A. et al. Systems modeling of the role of interleukin-21 in the maintenance of effector $\mathrm{CD}^{+}{ }^{+} \mathrm{T}$ cell responses during chronic Helicobacter pylori infection. mBio 5, e01243-14 (2014).

95. King, I.L. et al. A nonredundant role for IL-21 receptor signaling in plasma cell differentiation and protective type 2 immunity against gastrointestinal helminth infection. J. Immunol. 185, 6138-6145 (2010).

96. Pallikkuth, S. et al. Maintenance of intestinal Th17 cells and reduced microbial translocation in SIV-infected rhesus macaques treated with interleukin IL-21. PLoS Pathog. 9, e1003471 (2013).

97. Monteleone, G. et al. Interleukin-21 enhances T-helper cell type I signaling and interferon- $\gamma$ production in Crohn's disease. Gastroenterology 128, 687-694 (2005).

98. Stolfi, C. et al. Involvement of interleukin-21 in the regulation of colitisassociated colon cancer. J. Exp. Med. 208, 2279-2290 (2011).

99. Jauch, D. et al. Interleukin-21 controls tumor growth and tumor immunosurveillance in colitis-associated tumorigenesis in mice. Gut 60, 1678-1686 (2011).

100. Taylor, J.J. et al. Deletion and anergy of polyclonal B cells specific for ubiquitous membrane-bound self-antigen. J. Exp. Med. 209, 2065-2077 (2012).

101. Yang, Y. et al. Focused specificity of intestinal TH17 cells towards commensal bacterial antigens. Nature 510, 152-156 (2014).

102. Hepworth, M.R. et al. Innate lymphoid cells regulate $\mathrm{CD} 4^{+} \mathrm{T}$ cell response to intestinal commensal bacteria. Nature 498, 113-117 (2013).

103. Kawamoto, S. et al. T cells regulate immunoglobulin A selection and facilitate diversification of bacterial species responsible for immune homeostasis. Immunity 41, 152-165 (2014).

104. Ertelt, J.M. et al. Selective priming and expansion of antigen-specific Foxp3 ${ }^{-} \mathrm{CD}^{+}{ }^{+} \mathrm{T}$ Cells during Listeria monocytogenes infection. J. Immunol. 182, 3032-3038 (2009).

105. Lee, S.-J. et al. Temporal expression of bacterial proteins instructs host CD4 Tcell expansion and Th17 development. PLoS Pathog. 8, e1002499 (2012).

106. Pepper, M. et al. Different routes of bacterial infection induce long-lived TH1 memory cells and short-lived TH17 cells. Nat. Immunol. 11, 83-89 (2009).

107. Higgins, L.M. et al. Citrobacter rodentium infection in mice elicits a mucosal Th1 cytokine response and lesions similar to those in murine inflammatory bowel disease. Infect. Immun. 67, 3031-3039 (1999).

108. Higgins, L.M. et al. Role of bacterial intimin in colonic hyperplasia and inflammation. Science 285, 588-591 (1999).

109. Sinclair, J.F. \& O'Brien, A.D. Intimin types $\alpha, \beta$, and $\gamma$ bind to nucleolin with equivalent affinity but lower avidity than to the translocated intimin receptor. J. Biol. Chem. 279, 33751-33758 (2004). 